\title{
Pereutaneous Nephrolithotomy in Elderly Patients with Renal Calculi
}

\author{
Fan-ping MENG ${ }^{1}$, Jian-tao XIAN ${ }^{2}, \mathrm{Xu} \mathrm{ZHOU}^{3}$ and Ji GAO* \\ 1,2,3 Department of Urology, China-Japan Union Hospital Affiliated to Jilin University, \\ Changchun Jilin, 130033, China \\ * Department of Urology, China-Japan Union Hospital Affiliated to Jilin University, \\ Changchun Jilin, 130033, China
}

Keywords: Percutaneous nephrolithotomy (PCNL), Renal calculi, Elderly patients.

\begin{abstract}
Objective: To explore the safety and efficacy of percutaneous nephrolithotomy (PCNL) for the treatment of renal calculi in elderly patients. Methods: Totally 57 elderly patients (mean age 69 years, ranged from 61 to 87 years) with renal calculi were treated by PCNL in our hospital from January 2013 to December 2015. Working tunnels F16-F24 were established under B-ultrasound guidance. EMS or holmium laser lithotripsy was used to fragment and remove stones by nephroscopy. Results: The procedure was completed in a mean of 65 min (ranged from 30 to 140 rain), and one-session stone-free rate was $78.9 \%$ (45/57). After the procedure, one patient developed fever $\left(>38.5^{\circ} \mathrm{C}\right)$ and then was cured by antibiotics; two patients received blood transfusion( 1800 and $2500 \mathrm{ml}$, respectively)because of massive hemorrhage, and one of them was diagnosed with pseudoaneurysm, and then was cured by selective renal artery embolization. No patient had severe complications. Residual stones were detected in 12 patients, 4 of them received a second PCNL and 5 of them underwent postoperative ESML; one who received second PCNL and one who received ESML still showed a few residual stones in the lower renal calyces, the other 7 showed no residual stones after the second operation. Five of the 12 cases of residual stones were followed up for 6 to 24 months, 4 of them showed no significant change, and the other showed mildly enlarged renal stone. In the 46 patients who had the stones removed completely, follow-up was achieved for 3 to 34 months with a mean of 14.3 months, only 2 of them had recurrence and thus received conservative therapy. Conclusions: PCNL is safe and effective for elderly patients with renal stones. The keys of the therapy include adequate preoperative preparation, skilled operation, and perioperative management.

Elderly patients with heart and lung function compensatory ability are poor, and accompanied by chronic disease of other system. So the surgery is risking. Our hospital from January 2013 to December 2015, using percutaneous nephrolithotomy (PCNL) in treatment of 57 elderly kidney stones, the operation risk control and satisfactory effect is good, reports are as follows.
\end{abstract}

\section{The Methods and Data}

\section{The General Information}

57 cases are in group (male 36 cases and female 21 cases). The age of $61 \sim 87$ years old, average 69 years old. 29 cases of side waist pain, 2 patients have hematuria, and the other 26 cases of asymptomatic. Diagnosis of kidney stones by the CT: On the left side of the 32 cases, on the right side of 25 cases; single stone 19 cases, multiple 38 cases; stone diameter are $1.8 \sim 5.4 \mathrm{~cm}$, average $3.4 \mathrm{~cm}$. Light, medium and severe 
hydronephrosis of 16, 10, 2 cases respectively. .Renal insufficiency in 16 cases, serum creatinine are $168 \sim 369 \mu \mathrm{mol} / \mathrm{L}$ (normal value range $44 \sim 133 \mu \mathrm{mol} / \mathrm{L}$ ), 12 cases have urinary tract infection. 21 cases have the chronic disease, 14 cases have hypertension, 3 cases have coronary heart disease, 5 cases have chronic bronchitis, 8 cases have diabetes. 10 cases have extracorporal shock wave lithotripsy treatment 1 5 weeks ago, 8 cases with a surgery history of renal pelvis cut off and take the stone.

Case selection criteria: (1) age>60; (2) Kidney stone diameter $>2 \mathrm{~cm}$ or ESWL treatment failure; (3) No serious heart and lung function disorder and blood coagulation mechanism disorder.

\section{Methods}

Positive treatment of hypertension and diabetes, using sensitive antibiotics control urinary tract infection and lung infection, and improving heart and lung function. The patients that have kidney seeper with severe renal insufficiency, or difficult to control urinary tract infection, 2 cases of the retrograde indwelling double $\mathrm{J}$ tube, and 12 cases of them have the renal puncture drainage.

49 patients with general anesthesia, the rest 8 cases with lumbar anesthesia combined epidural anesthesia. Patients take the lithotomy position, through cystoscope or ureteroscope, at the side of the diseased ureter put in F5 F7 ureteral catheter, and indwelling catheter. 8 cases of cardiac insufficiency and older changes position to recumbent position, and the rest of the 49 cases take the prone position. Infusion pumps water injection forming artificial kidney seeper from the ureteral catheter. Piercing under No.11 or No.12 rib under axillary region puncture between posterior axillary line and linea scapularis, puncturing the right calyces successfully under the ultrasound, and exit the needle core and put the guide wire. Along the guide wire, we use fascia or metal expanders to expand the incision to F16 F24, leaving the corresponding working channel, and putting F8.0/9.8 ureteroscope or F20.8 percutaneous nephroscope in. We deal the renal calculi by $100 \mathrm{~W}$ holmium laser or EMS third-generation air pressure ballistic combined ultrasonic system. After making sure of no residual calculi, than indwell double $\mathrm{J}$ tube and nephrostomy tube. Review KUB to understand the presence of residual stone 3 5days after surgery, take ESWL or second PCNL surgery if it is necessary.

\section{Results}

The operation time is $30 \sim 140 \mathrm{~min}$, average time is $65 \mathrm{~min}$. Stone clearance rate is 78.9\% (45/57); 12 patients take a second PCNL or ESWL treatment 1 2 weeks after surgery. Postoperative have stone residues in 2 cases, residue stone diameter is $0.5 \sim$ $0.8 \mathrm{~cm} .1$ case of postoperative fever $>38.5^{\circ} \mathrm{C}$, improved after anti-infection treatment; 2 cases of intraoperative and postoperative bleeding 1800, $2500 \mathrm{ml}$, giving blood transfusion therapy. No damage to the liver, spleen and other important organs and induce respiratory and circulatory function failure and other serious complications. 5 cases calculi were followed up for 6 24 months, average time is 10.6 months, except 1 case of kidney stones slightly increases the rest 4 cases have no obvious change; 46 cases of calculus clean were followed up for 3-34 months, an average of 14.3 months, except 2 cases of conservative treatment of recurrence the rest without recurrence. 


\section{Discussion}

Elderly patients with heart and lung function compensatory ability are poor and often with chronic diseases, and have more risk getting PCNL. Preoperative control blood pressure, blood sugar, improve heart and lung function, make good important viscera function in the body and a steady state operation, in order to improve the surgical tolerance and reduce the risk of surgery.

Elderly patients with compensatory capacity decreased respiratory function is often characterized by functional residual capacity increase, ventilation and blood flow ratio imbalance, arterial blood oxygen partial pressure is reduced, less protective response to hypoxia and $\mathrm{CO}_{2}$ savings, respiratory protective hyporeflexia [1]. Elderly arteriosclerosis degree increased with increasing age, decreased neurohumoral factors adjust the function of blood vessels, vascular active substance produced and secreted decrease, adrenergic receptors fell, make regulating function decline, leading to blood rheology instability[2]. After anesthesia, circulation and breathing compensatory function further weaken, especially after the sudden change of position for the prone position easily induced acute circulation or respiratory insufficiency, especially in insufficient blood volume or lung dysfunction. General anesthesia surgery has good tolerance, and ensures the prone position in patients with airway open and oxygen supply, at the same time can be controlled through breathing or assistant breathing to reduce adverse impacts on the breath caused by change position. PCNL when patients take the prone position oppression abdomen bosom, obstruction the aorta and inferior vena cava diaphragmatic muscle activity and oppression, result in corresponding change of physiological functions such as breathing and circulation. Reclining position PCNL reduces the influence on breathing and circulation, improves the patient's ability to tolerate surgery, and reduces the risk of surgery [3]. 14\%(8/57) patients of this group take the reclining position PCNL, and the tolerance of the surgery is satisfacted.

Malhotra[4]et confirmed PCNL can exist in a large number of flushing fluid absorption, especially renal pelvis serious seeper or renal pelvis is tear. The elderly patients who heart and lung function is not complete and with poor circulation adjustment compensatory ability, along with the absorption intraoperative rinses, can appear the crisis such as acute pulmonary edema[5]. Also can appear complications such as dilution blood coagulation dysfunction, hypocalcaemia,hypoalbuminemia, hypokalemia and high chlorine acidosis[6]. Mohta[7] reported 20 cases of using physiological saline as perfusate PCNL adults, observing the postoperative blood $\mathrm{pH}$ value have obvious drop, bicarbonate in the blood values and negatively correlated with infusion duration, the number of percutaneous puncture and infusion duration and perfusion fluid absorption were positively correlated. In order to reduce the influence of flushing fluid absorption on the body, we summarized the experience in the following: (1) Percutaneous renal biopsy channel as the calyces fornices, can avoid large arteriovenous in kidney; (2) Reduce perfusion process water pressure and flow rate, shorten operation time; (3) EMS third-generation clear stone system makes intraoperative renal pelvis internal pressure drop even under negative pressure, reduce the liquid leakage[8]; (4) Delicate operation to avoid renal pelvis and calyces mucosal injury and calyces neck tearing; (5) Intraoperative strengthen the monitoring of hemodynamic and blood biochemistry, blood gas analysis, can be appropriately to give drugs such as diuretics and glucocorticoids when it is necessary.

At room temperature, large flushing fluid flows through the body can cause hypothermia, basal metabolic rate is low, and elderly patients during the operation of the application of anesthetic to further reduce the body heat output, and more easily 
influenced by ambient temperature. When patients significantly lower temperature can occur chills and tissue oxygen consumption increased, patients' heart rate quickens, increased blood pressure, decreased cardiac output, increase the risk in the elderly patients with cardiovascular disease [9]. The flushing fluid heating, which can effectively prevent PCNL perioperative hypothermia, chills, adverse reactions of heart rate and blood pressure changes, especially for make the elderly patients heart rate and blood pressure stable, has positive significance in reduce adverse reactions of heart, head, blood-vessel.

Elderly patients with chronic diseases, body comprehensive state on the low side, especially of diabetic patients with urinary tract infections is easy to have sepsis after PCNL. We confirmed that preoperative control blood sugar and control urinary tract infection by use sensitive antibiotics, adequate drainage in patients with kidney seeper when necessary, intraoperative take effective measures to reduce flushing fluid absorption, can avoid the occurrence of intraoperative and postoperative sepsis.

Elderly patients who have high blood pressure or diabetes with hardening of the arteries, blood coagulation dysfunction, renal dysfunction and urinary tract infection, is easily complicated have severe renal hemorrhage after PCNL. We have the following experience in prevention and treatment of severe bleeding in PCNL: (1) Preoperative 1 week discontinued anticoagulant drugs, correct blood coagulation dysfunction; (2) Preoperative positive control urinary tract infections; (3) Preoperative sufficient urine drainage to improve renal function who the obstruction to severe renal insufficiency; (4) The calyces dome puncture, when expand take the principle of light rather than deep; (5) Intraoperative soft operation, prevent the calyces neck tearing, reasonable arrangement of multiphase or multi-channel operation; (6) Elderly patients are more likely to cause disease of heart and head blood-vessel, serious bleeding after invalid conservative treatment should do the selective renal artery embolization as soon as possible.

\section{References}

[1] Yanjie Liu, Ning Liang, Li Ma, et al. Study of elderly patients with percutaneous nephroscope lithotripsy perioperative respiratory function according [J]. Guangxi Medicine, 2011, 33(7):807-809.In Chinese.

[2] Wenqiang Jiang. Low concentration ropivacaine epidural anesthesia in elderly patients with hip surgery. Jiangsu Medicine, 2006, 32:477-479.In Chinese.

[3] Yiping Zong, Weiming Zhou, Caixin Cao, et al. The reclining position percutaneous nephroscope treat urinary calculi. Chinese Journal of Minimally Invasive Surgery, 2011, 11(9):813-814.In Chinese.

[4] Malhotra S K, Khaitan A, Goswami A K, et al. Monitoring of irrigation fluid absorption during percutaneous nephrolithotripsy: the use of $1 \%$ ethanol as a marker[J]. Anaesthesia, 2001, 56(11):1103-6.

[5] Grove J J, Shinaman R C, Drover D R. Noncardiogenic pulmonary edema and venous air embolus as complications of operative hysteroscopy.[J]. Journal of Clinical Anesthesia, 2004, 16(1):48-50.

[6] Lixia Gan, Gang Liu, Yanjie Liu, et al. Researches of the change of blood gas analysis and hemodynamics in elderly patients with percutaneous nephrolithotomy. Guangxi Medicine, 2010, 32(8):917-918.In Chinese. 
[7] Mohta M, Bhagchandani T, Tyagi A, et al. Haemodynamic, electrolyte and metabolic changes during percutaneous nephrolithotomy.[J]. International Urology \& Nephrology, 2008, 40(2):477-82.

[8] Jianxin Li. EMS three generations of rubble stone qing system in the application of percutaneous nephroscope lithotripsy. Chinese Journal of Urology, 2008, 29(10):659.In Chinese.

[9] YE Min, Jianhua CHEN, Jian KANG, et al. Effects of the temperature of irrigation fluid on body temperature and cardiovascular performance during transurethral surgery. Chin J Urol, 2002, 23(7):417-418.In Chinese. 